Original article

HNO $2021 \cdot 69$ (Suppl 1):S7-S19

https://doi.org/10.1007/s00106-020-00919-9

Published online: 12 October 2020

(c) The Author(s) 2020

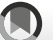

Check for
updates

S. K. Plontke ${ }^{1}$ P. Caye-Thomasen ${ }^{2} \cdot$ C. Strauss ${ }^{3} \cdot$ S. Kösling ${ }^{4} \cdot$ G. Götze ${ }^{1}$.

U. Siebolts ${ }^{5} \cdot$ D. Vordermark ${ }^{6} \cdot$ L. Wagner ${ }^{1} \cdot$ L. Fröhlich ${ }^{1} \cdot$ T. Rahne $^{1}$

'Department of Otorhinolaryngology, Head \& Neck Surgery, Martin Luther University Halle-Wittenberg, University Medicine Halle, Halle (Saale), Germany

${ }^{2}$ Department of Oto-Rhino-Laryngology, Head and Neck Surgery, Copenhagen University Hospital Rigshospitalet, Copenhagen, Denmark

${ }^{3}$ Department of Neurosurgery, Martin Luther University Halle-Wittenberg, University Medicine Halle, Halle, Germany

${ }^{4}$ Department of Radiation Medicine, Clinic for Radiology, Martin Luther University Halle-Wittenberg, University Medicine Halle, Halle, Germany

${ }^{5}$ Institute of Pathology, Martin Luther University Halle-Wittenberg, University Medicine Halle, Halle, Germany

${ }^{6}$ Department of Radiation Medicine, Clinic for Radiotherapy, Martin Luther University Halle-Wittenberg, University Medicine Halle, Halle, Germany

\title{
Management of transmodiolar and transmacular cochleovestibular schwannomas with and without cochlear implantation
}

\section{Video online}

The online version of this article (https:// doi.org/10.1007/s00106-020-00919-9) includes a video (2D and 3D versions) of the described surgical technique. Article and supplementary material are available at www.springermedizin.de. Please enter the title of the article in the search field, the supplementary material can be found under "Ergänzende Inhalte".

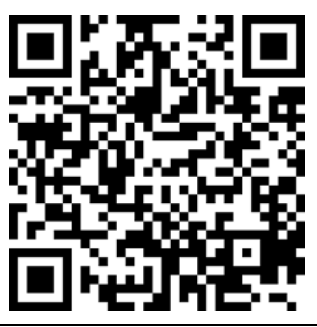

\section{Introduction}

Cochleovestibular schwannomas are benign tumors of the eighth cranial nerve with an incidence of approximately $3.4 / 10^{5}$ [41]. They usually originate from the vestibular nerve and occur as uni- lateral, sporadic, nonsyndromic tumors. Bilateral occurrence has been observed in $5-10 \%$ of cases associated with neurofibromatosis 2 (NF2) [12]. The usual tumor locations are the internal auditory canal (IAC) and the cerebellopontine angle (CPA) [27]; however, they can also occur as intralabyrinthine schwannomas (ILS) in the terminal branches of the eighth cranial nerve in the inner ear (recently reviewed in [8]).

Hearing loss because of vestibular schwannomas (VS) negatively influences the quality of life and patients with this condition are increasingly interested in hearing rehabilitation with cochlear implantation (CI) $[25,28,57]$. Several case reports, case series and first systematic reviews have illustrated that after surgical removal of sporadic VS or VS associated with NF2, CI can lead to good hearing results, even though not all patients will achieve good speech understanding. The CI may be performed in one stage with tumor removal $[3,45,50,57]$ or in a second surgery $[3,4,17,22,42]$. Similar initial results for hearing rehabilitation are available for CI after radiotherapy $[9,28,31]$ and with a wait and test and scan strategy [5]. For ILS limited to the inner ear, surprisingly good results with CI with respect to speech understanding have been reported, despite substantial cochlear trauma from the surgical tumor removal [2, 35, 36, 38].

Special subtypes of cochleovestibular schwannomas are located in the inner ear and the IAC, and sometimes the CPA, i.e., transmodiolar (TMOD, cochlea and IAC), transmacular (TMAC, vestibule and IAC), or translabyrinthine (TLab, intravestibulocochlear with TMOD and TMAC growth into the IAC) (• Table 1, - Fig. 1). For TMOD and TLab schwan-

The German version of this article can be found under https://doi.org/10.1007/s00106020-00918-w.

The manuscript is part of the special issue "Prize winners of the German Society of Otorhinolaryngology, Head and Neck Surgery." It contains data presented at the 90th Annual Meeting of the German Society of Otorhinolaryngology, Head and Neck Surgery 2019 [34], where it was awarded the "1st poster prize in the category clinical research." 


\section{Original article}

Table 1 Classification of cochleovestibular schwannomas with location in the inner ear and the internal auditory canal. (After Kennedy et al. [21] and van Abel et al. [54])

\begin{tabular}{|c|c|c|c|c|c|c|}
\hline Classification & Abbreviation & Cochlea & Vestibule \pm SCC & IAC & Middle ear & CPA \\
\hline Transmodiolar $^{\mathrm{a}}$ & TMOD & $X$ & $(X)$ & $X$ & - & - \\
\hline Transmacular ${ }^{b}$ & TMAC & - & $x$ & $X$ & - & - \\
\hline Translabyrinthine $^{c}$ & TLab & $x$ & $X$ & $x$ & - & - \\
\hline Transotic & TO & $x$ & $x$ & $x$ & $x$ & - \\
\hline Involvement of the CPA & $+\mathrm{CPA}$ & \pm & \pm & $X$ & \pm & $X$ \\
\hline \multicolumn{7}{|c|}{$\begin{array}{l}\text { 'Extension through the modiolus into the internal auditory canal } \\
\text { 'Extension through the macula cribrosa into the internal auditory canal } \\
\text { 'Extension through the modiolus and the macula cribrosa into the internal auditory canal. For intravestibulocochlear tumors and location in the fundus } \\
\text { of the IAC, the differentiation to transmacular and transmodiolar extension might be difficult. In our case series no tumor with certain transmodiolar and } \\
\text { transmacular (i.e., translabyrinthine) extension was present. Also, this type of tumor extension is not part of the Kennedy et al. classification [21] } \\
\text { IAC internal auditory canal, CPA cerebellopontine angle, SSC semicircular canals }\end{array}$} \\
\hline
\end{tabular}

a

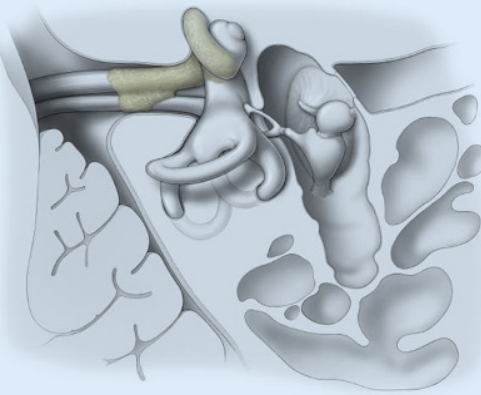

b

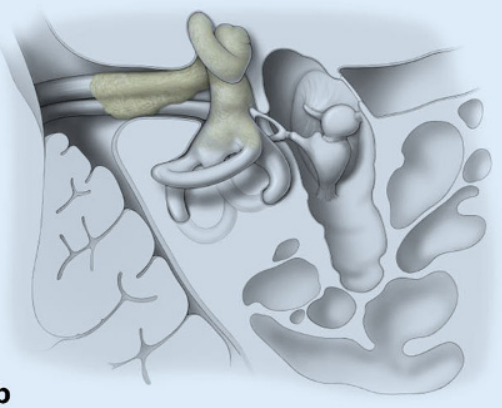

c

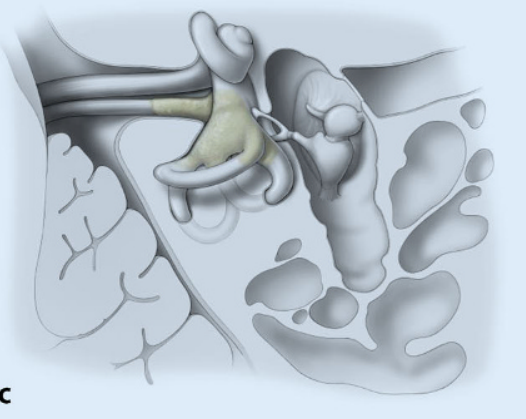

Fig. $1 \Delta$ Schematic drawings of tumor extensions from the inner ear to the internal auditory canal. a Intracochlear schwannoma with transmodiolar extension. b Intravestibulocochlear schwannoma with transmodiolar extension. c Intravestibular schwannoma (+ partially semicircular canals) with transmacular extension. Additional growth into the cerebellopontineangle is also possible (not shown). The facial nerve is located cranially to the cochlear nerve and thus not shown. For simplification, only one vestibular nerve is shown. (Artist: Hans Jörg Schütze, Medical Illustrator, Köln, Germany)

nomas, CI is possible (and meaningful) only with incomplete tumor removal because complete removal would also include destruction of the spiral ganglion cells in the modiolus, which are necessary for electrical stimulation through the implant. First results have been promising in patients for whom hearing rehabilitation was a high priority, including CI without intracochlear tumor removal [6] or with partial removal of the intracochlear parts of the tumor and reduction of the retrocochlear portion only (IAC + CPA) [39].

Here, we report our experiences with the surgical management of cochleovestibular schwannomas with TMOD or TMAC extension and hearing results after CI.

\section{Methods}

For this retrospective analysis, patients with a TMOD, TMAC, TLab, or transotic (TO) extension of cochleovestibular schwannoma were included (classification after [21, 54]; - Table 1). Patients were selected from a consecutive personal case series of the first author at a tertiary (university) referral center with an interdisciplinary skull base center. The tumors were either primary ILS or classical (intrameatal) schwannomas with secondary invasion of the inner ear and with or without extension into the CPA. Patients were treated between November 2013 and April 2020 and underwent either complete or partial tumor removal with or without CI.

Depending on tumor location and extension, surgery involved a translabyrinthine/transotic approach to the IAC and the CPA or a transmeatal partial or subtotal cochleoectomy (for details see - Table 2 and "Results"; [2, 33, 35, 36, 38, 39]).

Selection of the type of cochlear implant and electrode array was based on experience and audiological results with previous surgery for ILS [35-38]. In addition, we ascribed to the hypothesis that a perimodiolar electrode array position with close contact of the electrode pads to the spiral ganglion cells in Rosenthal's canal (here: CI512, CI612, CMD electrodes) with cartilage and fibrosis lateral to the array will lead to reduced spread of the electric field, contributing to a good hearing result after partial and subtotal cochleoectomy [56]. Furthermore, the improved magnetic resonance imaging (MRI) compatibility of the magnets in the receiver coil for easier MRI followup of the residual tumor was considered, which at that time was mainly present for a limited number of implant models 
(e.g., Synchrony FLEXSOFT, MED-EL, Innsbruck, Austria and Synchrony CMD, MED-EL, later also CI612, Cochlear, Sydney, Australia; - Table 2).

Because of the small number and heterogeneity of patients with this rare tumor entity, statistical evaluation was mainly descriptive with a focus on surgical management and adverse events. In patients who underwent CI, the primary endpoint was word recognition in quiet conditions (Freiburger multisyllabic numbers and monosyllables at $65 \mathrm{~dB}$ SPL), with masking of the contralateral ear.

Secondary outcome parameters were intraoperative, electrically evoked (via the implant) compound action potentials (eCAP), impedance measures, the presence of a wave $\mathrm{V}$ in intraoperative and postoperative electrically evoked brainstem potentials (eABP), and characteristics during implant fitting and programming.

The eCAP were measured with the settings included in the respective manufacturer's implant programming software (AutoNRT or AutoART). In patients who received a Nucleus implant, a transmodiolar stimulus was used for the eABP [11, 39]. The same stimulus was also used for recording of additional eCAP (AdvancedNRT). In patients with Synchrony implants, individual electrodes or electrode clusters distributed along the array were stimulated.

\section{Results}

From a total of 53 consecutive patients with ILS, 9 had tumors with TMOD $(n=6)$, TMAC $(n=2)$ or TO $(n=1)$ extension. Involvement of the CPA (+CPA) was found in three patients (• Figs. 2, 3 and 4). Demographic and baseline data, tumor location, duration of hearing loss before surgery, surgical management, and if appropriate, hearing results with CI are shown in - Table 2. All patients were informed of the different management options: 1) wait and test and scan, 2) radiotherapy, 3) complete or incomplete tumor removal, and 4) if possible, hearing rehabilitation with CI. None of the patients chose radiotherapy. Patient 3 was observed until the hearing threshold deteriorated, a hear-

HNO 2021 - 69 (Suppl 1):S7-S19 https://doi.org/10.1007/s00106-020-00919-9

(c) The Author(s) 2020

S. K. Plontke · P. Caye-Thomasen · C. Strauss · S. Kösling · G. Götze · U. Siebolts · D. Vordermark · L. Wagner $\cdot$ L. Fröhlich $\cdot$ T. Rahne

\section{Management of transmodiolar and transmacular cochleovestibular schwannomas with and without cochlear implantation}

\begin{abstract}
Introduction. Hearing rehabilitation with cochlear implants has attracted increasing interest also for patients with cochleovestibular schwannoma. The authors report their experience with the surgical management of tumors with rare transmodiolar or transmacular extension and outcomes after cochlear implantation $(\mathrm{Cl})$.

Methods. This retrospective case series included nine patients with either primary intralabyrinthine tumors or secondary invasion of the inner ear from the internal auditory canal. The primary endpoint with $\mathrm{Cl}$, performed in six patients, was word recognition score at $65 \mathrm{~dB}$ SPL (sound pressure level). Secondary endpoints were intra- and postoperative electrophysiological parameters, impedance measures, the presence of a wave $V$ in the electrically evoked (via the $\mathrm{Cl}$ ) auditory brainstem responses, the specifics of postoperative $\mathrm{Cl}$ programming, and adverse events.

Results. Hearing rehabilitation with $\mathrm{Cl}$ in cases of transmodiolar tumor growth could be achieved only with incomplete tumor
\end{abstract}

ing aid could not be tolerated because of a reduced uncomfortable loudness threshold, and symptoms of vertigo developed. Patient 5 with NF2 and bilateral deafness was observed until the ipsilateral residual tumor after partial tumor resection had remained stable for some years. The two patients with transmacular tumor extension (8 and 9) showed fluctuating hearing loss, likely because of a secondary endolymphatic cochlear hydrops detected on MRI (• Fig. 5a, c). Patient 9 reported increasing dizziness.

Tumor removal was done by previously described surgical techniques either completely (patients 1, 2, 8, and 9; - Fig. 2a-d and 5) or incompletely (patients 3-7; - Fig. 2f, 3 and 4). Surgical removal of the TO tumor through a translabyrinthine/transotic approach and a lateral petrosectomy (patient 1; - Fig. 2a, b) also has been described before [38]. In the two patients with removal, whereas tumors with transmacular growth could be completely removed. All six patients with $\mathrm{Cl}$ had good word recognition scores for numbers in quiet conditions (80-100\% at $65 \mathrm{~dB}$ SPL, not later than 6 to 12 months post $\mathrm{Cl}$ activation). Four of these six patients achieved good to very good results for monosyllabic words within $1-36$ months (65-85\% at $65 \mathrm{~dB}$ SPL). The two other patients, however, had low scores for monosyllables at 6 months ( 25 and $15 \%$ at $65 \mathrm{~dB}$ SPL, respectively) with worsening of results thereafter.

Conclusions. Cochleovestibular schwannomas with transmodiolar and transmacular extension represent a rare entity with specific management requirements. Hearing rehabilitation with $\mathrm{Cl}$ is a principal option in these patients.

\section{Keywords}

Evoked potentials, auditory, brain stem . Schwannoma, intralabyrinthine - Neuroma, acoustic $\cdot$ Ear, inner $\cdot$ Rehabilitation
TMAC extension from the vestibule along the superior vestibular nerve into the IAC, tumor removal was done via a translabyrinthine approach, preserving the cochlear nerve and the cochlea (- Fig. 5d-h). Incomplete tumor removal was carried out by a transmeatal partial or subtotal cochleoectomy [33, $35,36,38]$ or via push-through or pullthrough techniques (also called pipe cleaner, beach towel, or dental floss techniques) $[2,26,36,39]$. The video (2D and $3 \mathrm{D}$ versions, see $\mathrm{QR}$ code) shows the transmeatal surgical removal of the intracochlear tumor parts through a partial cochleoectomy and $\mathrm{CI}$ in the patient with NF2.

Postoperatively, two patients (patients 7 and 9) reported temporary moderate vertigo. Another patient (patient 3) experienced continuation of a pre-existing vertigo for some weeks after surgery, but the condition continuously improved 


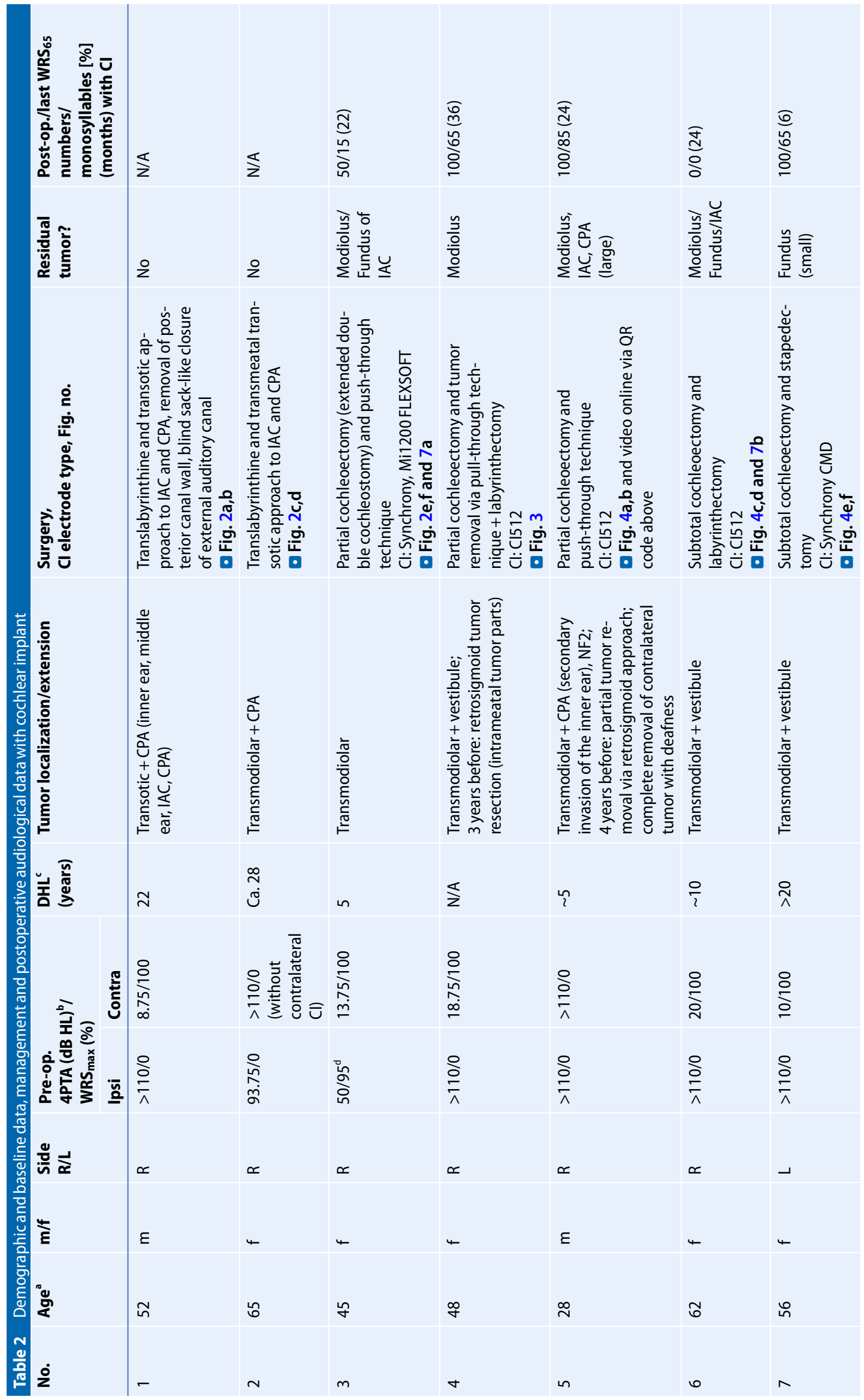




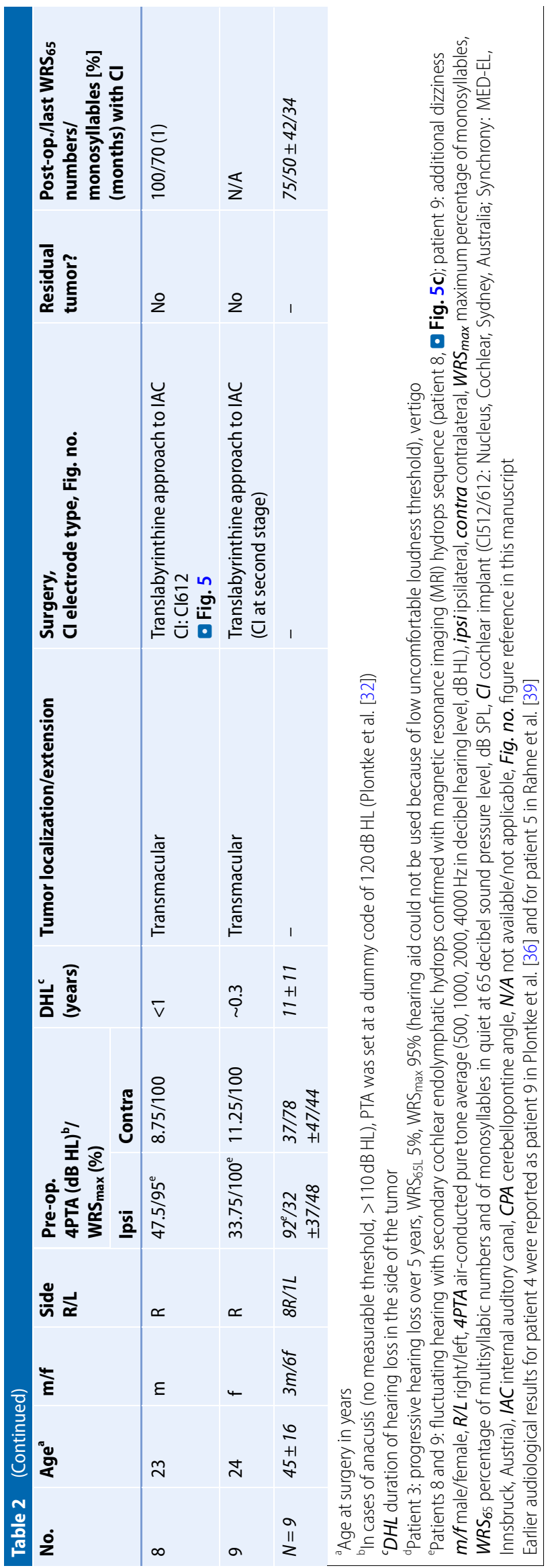

during the first postoperative year. In patient 2 a temporary incomplete facial paralysis (House-Brackmann II-III) developed but resolved completely within a couple of months. There were no severe adverse events.

Five patients received an implant with a perimodiolar electrode array, either with a Contour Advance electrode (Nucleus CI512, $n=3$; Nucleus CI612, $n=1$; Cochlear) or as a custom-made device (Synchrony Mi1200 CMD, MEDEL, $n=1$ [37]). One patient received an implant with a lateral wall electrode array (Synchrony Mi1200 FLEXSOFT, MED-EL).

Intraoperatively, an increased impedance in one electrode was found in two patients. In all other patients, electrode impedances were homogeneous and below $15 \mathrm{k} \Omega$. The intraoperative recording of eCAP (AutoNRT or AutoART) showed measurable thresholds for $1-5$ electrodes in 3 patients. All electrodes showed measurable eCAP thresholds only in patient 8 .

Postoperative audiological results with the implant are listed in D Table 2 (last available measurement) and are shown individually over time in $\mathbf{0}$ Fig. 6. All patients had good word recognition scores at $65 \mathrm{~dB}\left(\mathrm{WRS}_{65}\right)$ for multisyllabic numbers in quiet conditions $(80-100 \%$ at $65 \mathrm{~dB}$ SPL, not later than 12 months after first fitting). Of the six patients who underwent $\mathrm{CI}$, four reached good to very good $\mathrm{WRS}_{65}$ for monosyllables (65-85\% at $65 \mathrm{~dB}$ SPL). Two patients had only poor $\mathrm{WRS}_{65}$ after 6 months (patient 3: $25 \%$, and patient 6: $15 \%$ at $65 \mathrm{~dB}$ SPL), with further worsening of results thereafter. The follow-up MRIs in these two patients showed only minimal tumor growth of $0.5 \mathrm{~mm}$ (2 years after implantation) in patient 3 (• Fig. 7a), and growth of $3.6 \mathrm{~mm}$ (2 years and 5 months after implantation) in patient 6 (• Fig. 7b).

On average, patient $\mathrm{WRS}_{65}$ values were $94 \pm 9 \%$ for numbers and $52 \pm 31 \%$ for monosyllables at $65 \mathrm{~dB}$ SPL (after 6 months, $n=5$ ). Twelve months after the first fitting of the audio processor, data were available for three patients (patients 4, 5, and 6), and the $\mathrm{WRS}_{65}$ was $93 \pm 12 \%$ for numbers and $57 \pm 45 \%$ for monosyllables. 


\section{Original article}
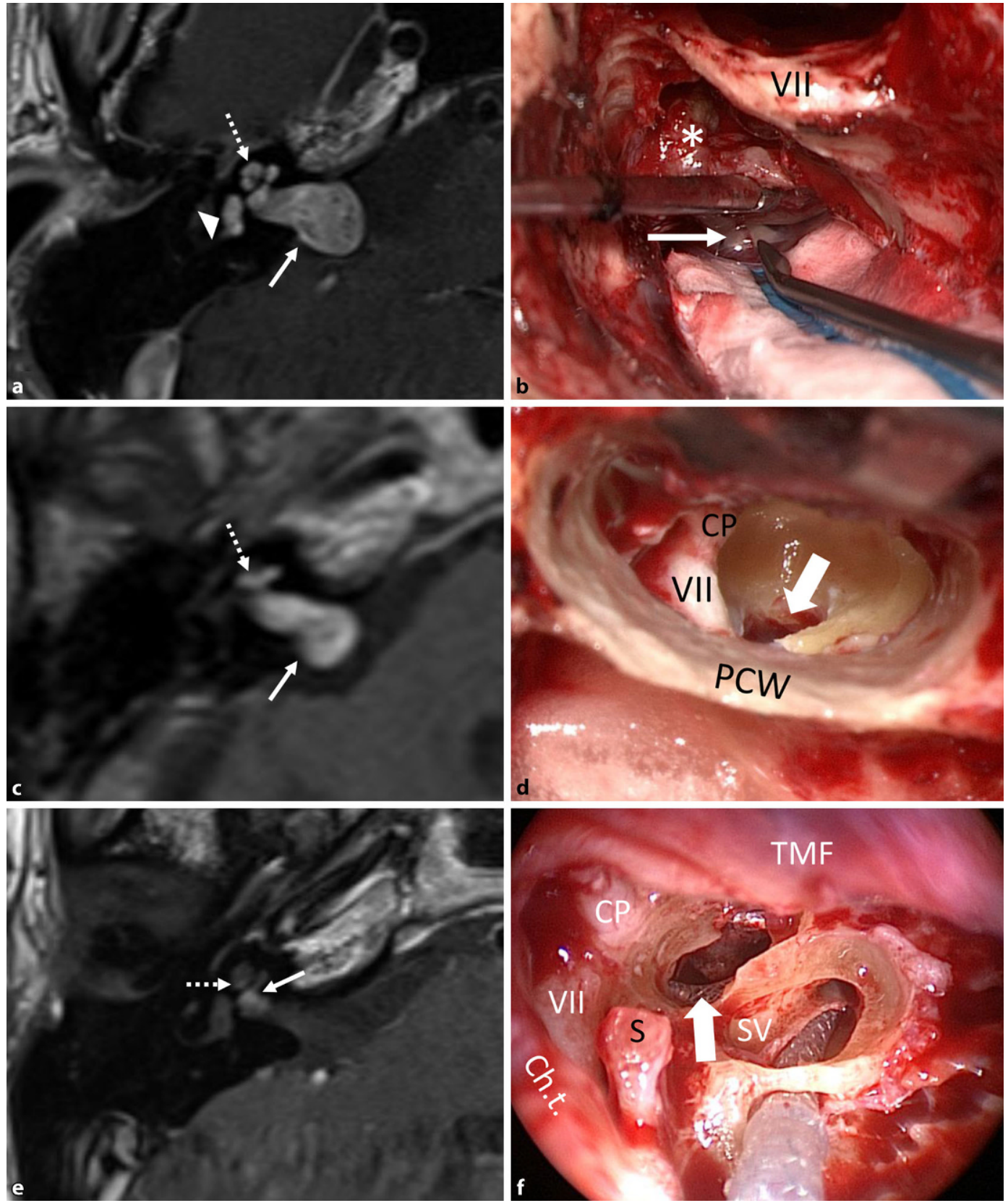

Fig. $2 \Delta$ a,b Patient 1 in $\bullet$ Table 2. a Magnetic resonance imaging (MRI, axial, T1-w + gadolinium, Gd) showing the transotic tumor with typical gadolinium enhancement in the entire inner ear, IAC and CPA $(\rightarrow)$, (middle ear extension not shown). b Intraoperative picture showing the facial nerve canal in the mastoid (VII) after lateral petrosectomy and tumor (asterisk) in the IAC + CPA during separation from the facial nerve $(\rightarrow)$. c, d Patient 2 in 0 Table 2. c MRI (axial, T1-w + Gd) showing the tumor in the cochlea and in the IAC +CPA $(\rightarrow)$. $\mathbf{d}$ Intraoperative view of the fundus of the IAC $(\rightarrow)$ after combined translabyrinthine and transmeatal-transotic tumor resection. e, f Patient 3 in - Table 2. e MRI (axial, T1-W+Gd) showing a transmodiolar ILS with tumor in the second turn of the cochlea and extension into the fundus of the IAC $(\rightarrow)$. f Intraoperative view after tumor resection from the cochlea with the implant electrode array at the lateral cochlear wall $(\rightarrow)$. The basal turn was free of tumor VII facial nerve, Ch.t. chorda tympani, $C P$ cochleariform process; $C P A$ cerebellopontine angle, Gd gadolinium, IAC internal auditory canal, $P C W$ posterior canal wall, TMF tympanomeatal flap, $S$ stapes head, SV scala vestibuli, $w$ weighted. Dotted arrow tumor in cochlea, triangle tumor in vestibule 

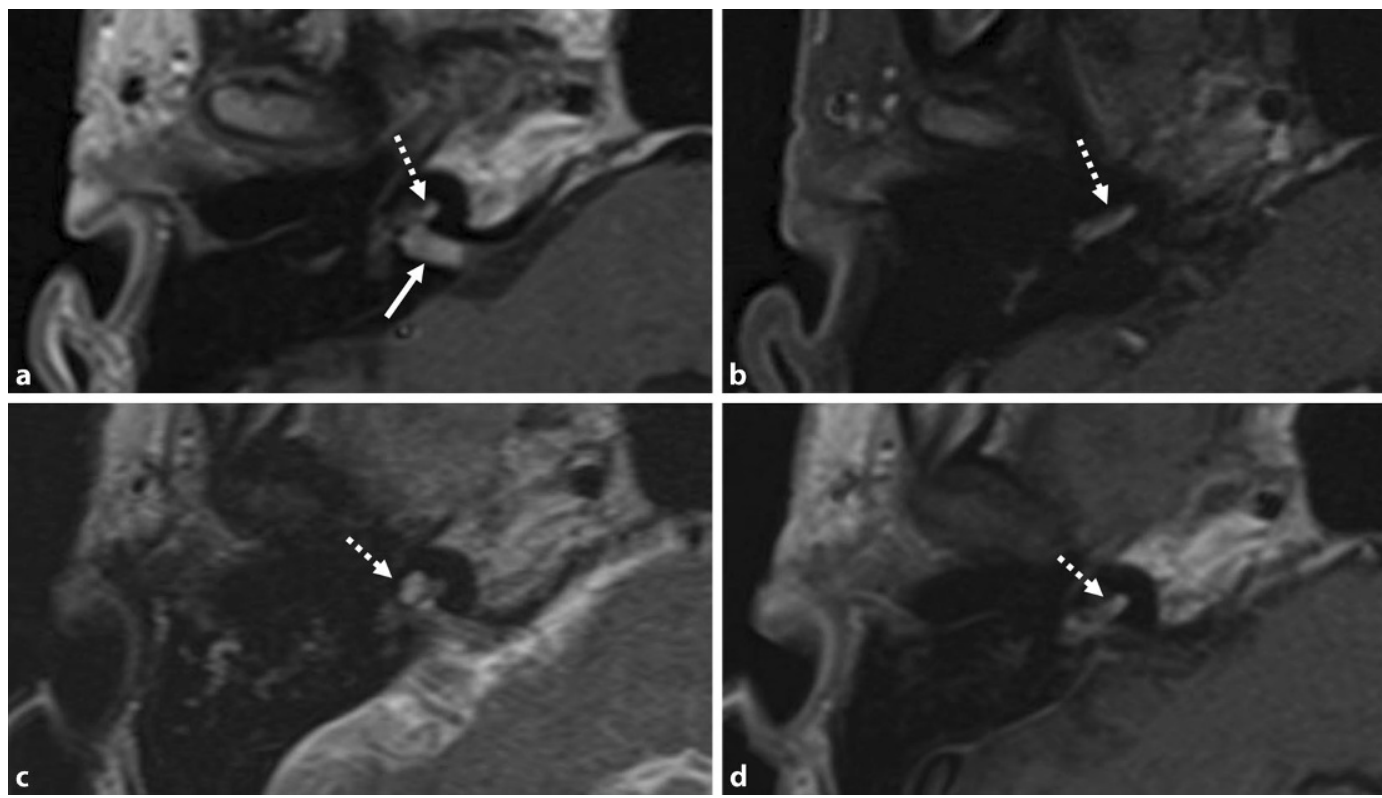

Fig. $3 \varangle$ Patient 4 in

- Table 2. MRI (axial,

T1-w, + Gd). Transmodiolar ILS with resection of the intrameatal and, later, the intracochlear tumor parts. a,b Schwannoma in the IAC $(\rightarrow)$ and missed tumor in the cochlea (dotted arrows; 2013). c,d Situation 3 years after neurosurgical resection of the intrameatal and before resection of the intracochlear tumor parts

Postoperative impedances were unstable in four patients and varied over time. In patient 5 , a functional short circuit between two electrodes was found after 6 months. The other patients had stable, homogeneous impedances below $15 \mathrm{k} \Omega$.

The eABP data were available for four patients. In three patients with a $\mathrm{Nu}$ cleus CIx12 implant, a wave V could be recorded at thresholds of 160-180 current units (cu). In two patients, this correlated with eCAP thresholds that were recorded with the same stimulus. In patient 7, who had the Synchrony CMD implant, no eABP thresholds could be measured.

Postoperative implant programming could be done in all patients based on subjective information through loudness scaling. In 3 patients, 2-4 electrodes had to be deactivated because of discomfort or missing loudness growth. In four patients, relatively high charge levels with an increased pulse width were necessary.

\section{Discussion}

This case series presents our experiences with the surgical management of a rare subgroup of cochleovestibular schwannomas, including hearing rehabilitation with cochlear implant in some of these patients. In two patients with $\mathrm{TO}$ or TMOD tumor extension including the CPA (patient 1, - Fig. 2a, b, and patient 2,
- Fig. 2c, d), complete tumor removal including the intramodiolar tumor parts had priority for the patients over hearing rehabilitation; thus, CI was not possible. In the two patients (8 and 9) with TMAC tumor growth, the tumor could be completely removed through a translabyrinthine approach with preservation of the cochlear and facial nerves (• Fig. 5). Already at the first fitting, the patient with TMAC who underwent CI had reached surprisingly good $\mathrm{WRS}_{65}$ for monosyllables $(70 \%$ at $65 \mathrm{~dB}$; - Table 2, - Fig. 6). The five patients with incomplete tumor removal showed a larger variance in $\mathrm{WRS}_{65}$ for monosyllables (minimum 0\%, maximum: 100\%; • Fig. 6). The mean $\mathrm{WRS}_{65}$ for monosyllables after 6 months (52\%) was still similar to results for other patients having CI [18, 24, 43], especially compared to patients with implants for single-sided deafness $[1,13$, $16,40,55]$. These results are also similar to or better than those reported in small case series with CI after translabyrinthine resection of classical VS (IAC \pm CPA) [4, $17,22,42,57]$. The $\mathrm{WRS}_{65}$ values, however, were not as good and consistent as those reported for patients with CI after removal of solely intracochlear and intravestibulocochlear schwannomas [35, $36,56]$. There were no serious adverse events.

Intraoperative eCAP and intraoperative and/or postoperative eABP were recorded as secondary outcome parameters. Although for preoperative eABP measurements only the promontory can be stimulated, the intracochlear implant electrode was used as an optimal stimulation site for intraoperative and postoperative recordings. The recordings in patient 7 (CMD electrode) were performed together with the manufacturer. No wave $\mathrm{V}$ could be detected in this patient, most likely because of suboptimal stimulation parameters. In all other patients who had a CI, a clear wave V could be recorded. Although all patients (including patient 7) achieved an auditory impression, word recognition ranged from very good (patient 5) to very poor (patient 6). Thus, eABP measurements in principle correlated with implant function, but without a correlation with the expected word recognition. Similar conclusions can be made for the eCAP with preset stimulation parameters, which could be recorded for all electrodes in only one patient.

These observations also correlated with the CI programming. Electrodes had to be deactivated in 3 out of 6 patients, and charge levels had to be set relatively high in 4 out of 6 patients. The fluctuation impedances indicate intracochlear changes, which might explain the deterioration in speech understanding that some of the patients experienced. Whether these changes are the result of 


\section{Original article}
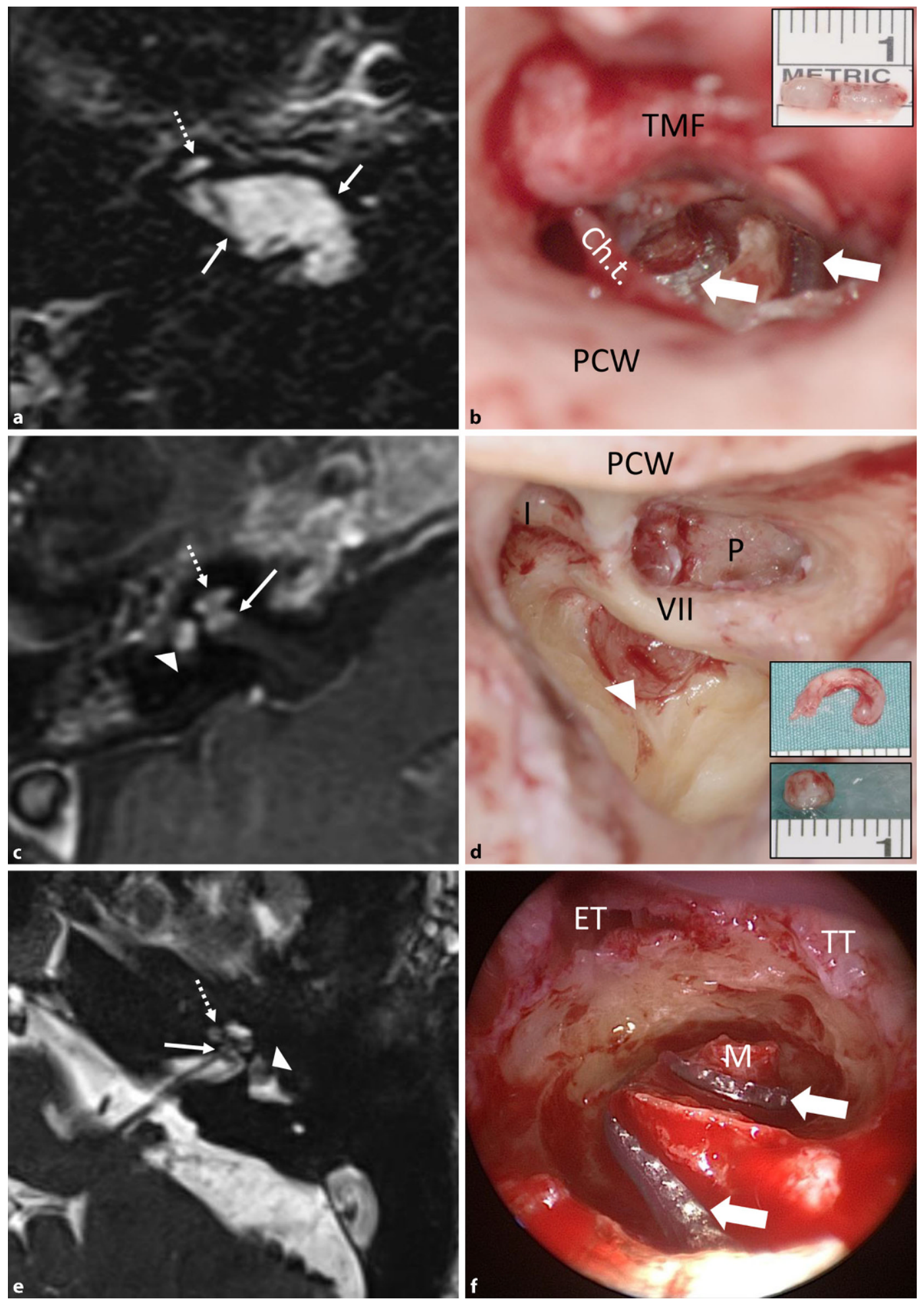
Fig. $4 \triangleleft$ a,b Patient 5 in $\bullet$ Table 2. a MRI (axial, $\mathrm{T} 1-\mathrm{W}+\mathrm{Gd}$ ) showing the schwannoma in the IAC + CPA $(\rightarrow)$ with secondary, transmodiolar extension in the cochlea in a patient with neurofibromatosis 2 after partial tumor resection via a retrosigmoid approach. The residual tumor was stable during a 4-year observation period. $b$ Intraoperative view with the perimodiolar electrode array in the first and second cochlear coils after intracochlear tumor resection (insert picture). The modiolus was completely preserved (Rahne et al. [39]). The surgical technique is illustrated in the intraoperative video (2D, 3D). c, d Patient 6 in 0 Table 2. c MRI (axial, T1-w + Gd) showing the tumor in the cochlea, in the vestibule, and in the fundus of the IAC. $\mathbf{d}$ Intraoperative view with tumor in the vestibule. Upper inset image: intracochlear tumor parts, removed through a transmeatal, subtotal cochleoectomy. Lower inset picture: intravestibular tumor parts. e, f Patient 7 in - Table 2. e MRI (axial, T2-w) showing an intravestibulocochlear ILS with transmodiolar extension into the fundus of the IAC $(\rightarrow)$ as a missing fluid signal. $f$ Intraoperative view with a perimodiolarimplant electrode array (custom-made device, $\rightarrow$ ) after tumor resection with preservation of the first and second turn modiolus. VII facial nerve, I incus body, Ch.t. chorda tympani, $E T$ eustachian tube, Gd gadolinium, IAC internal auditory canal, $P$ promontory; $P C W$ posterior canal wall, $T M F$ tympanomeatal flap, $T T$ tensor tympani muscle, $w$ weighted. Dotted arrow tumor in cochlea, triangle tumor in vestibule

increasing fibrosis or residual tumor tissue could not be determined.

The limitations of the presented observations lie in the small number and heterogeneity of the patient group and the relatively short follow-up period (maximum 36 months). The ILS are rare tumors, and the patients in this case series are a subgroup, representing only $17 \%$ (9 out of 53) of our overall cohort of ILS patients. Other authors have reported this subgroup of tumor locations and extensions (TMOD, TMAC, TO, and TLab) in $8 \%$ [54], 17\% [10], 29\% [21], and $42 \%$ [44] of their respective case series.

We note that in an individual case, it often cannot be determined whether the tumor is a primary ILS (arising from the inner ear) or started from the IAC with secondary invasion into the inner ear (invasive schwannoma [27]). Initial imaging results frequently already show the tumor in both the inner ear and the fundus of the IAC. An exception in our case series was the patient with NF2 (patient 5). Initial MRI showed the tumor in the IAC and the CPA but not in the inner ear (suggesting secondary ILS or invasive schwannoma). In patients 2 , 3 , and 4 , the origin of the tumor was assumed to be in the IAC. In view of missing earlier MRI images, however, we could not be certain. In addition, a differentiation between TLab and TMOD/ TMAC tumor extension is often difficult if the tumor is located in the cochlea and the vestibule ( \pm semicircular canals) and completely fills the fundus of the IAC (e.g., patient 6, ๑ Fig. 4c). For surgical management, however, the differentiation between primary versus secondary and TMOD versus TLab does not play a significant role. In cases of tumor extension through the modiolus, hearing rehabilitation with a CI is not compatible with complete tumor removal because spiral ganglion cells in the modiolus are necessary for electrical stimulation. The TMAC tumors represent a special situation, at least with limited extension into the IAC. In these cases, complete tumor removal with preservation of the cochlear nerve in the IAC and very good results for speech understanding with implants seem to be possible (patient 8; - Figs. 5 and 6, - Table 1).

In addition to surgery, radiotherapy is another treatment option; however, the proximity of the sensory or ganglion cells to the target region needs to be considered. Radiotherapy arrests the tumor growth of classical (IAC $\pm \mathrm{CPA}$ ) VS. Initial reports for radiotherapy for VS (no ILS) and CI showed positive results for hearing rehabilitation [5, 9, 31]. Although data for the tolerance doses of the various inner ear structures are sparse, even the frequently recommended cochlear tolerance doses of approximately $5 \mathrm{~Gy}$ for single-fraction radiosurgery (SRS) and $35 \mathrm{~Gy}$ for fractionated stereotactic radiotherapy (FSRT) are difficult to achieve in any inner ear location, given the required therapeutic doses of approximately $12 \mathrm{~Gy}$ in SRS and $54 \mathrm{~Gy}$ in FSRT [30]. Irrespective of the radiotherapy technology (SRS via gammaknife or cyberknife, FSRT via linear accelerator), deterioration of existing hearing ability (e.g. cases \#3, \#8, \#9) and restrictions for later rehabilitation with CI are expected.
Even a CI without a therapeutic intervention with respect to the tumor (implant + wait and test and scan) appears to be possible. The first results in patients with intrameatal tumors ( \pm CPA) were promising [5]. In patients with a tumor in the inner ear, however, the implant electrode array would need to be inserted through the tumor, as described by Carlson et al. [6].

After gross total tumor resection (patients 1, 2, 8, and 9 in $\bullet$ Table 1), recommendations for MRI follow-up are similar to those for resection of intrameatal ( \pm CPA) tumors. No specific recommendations exist for patients with possible tumor remnants in the modiolus (e.g., after resection of intracochlear, intravestibular, and intravestibulocochlear schwannomas through a subtotal cochleoectomy $[35,36])$ and/or labyrinthectomy or with only small remnants in the modiolus (e.g., patient 4) or in the fundus of the IAC (e.g., patient 7). Any presence of residual tumor bears the significant risk of growth of the remaining tumor tissue. Even after gross total resection, however, long-term follow-up with MRI is necessary, which patients explicitly need to be told. In a retrospective study, 52 out of $396(13 \%)$ patients with gross total tumor resection experienced a recurrence after a mean of 7.5 years [29]. In contrast, after incomplete but near total resection of intrameatal tumors ( \pm CPA), most patients experienced no or no relevant tumor recurrence. In a series of 1143 patients with translabyrinthine removal of VS evaluated intraoperatively as total $(n=978)$, near total $(n=140)$, or subtotal $(n=25)$ tumor removal, Hahn et al. (2013) reported a rate of revision surgery of $1.2 \%$, i.e., 14 patients with 2 initially receiving total, 5 near total, and 6 subtotal excisions. The authors concluded that most residual tumors disappear spontaneously, probably due to devascularization [15]. Other groups have reported similar observations. The authors noted that the growth rate of residual tumors is less for near total than for subtotal resection and that long-term MRI follow-up ( 10 years) is necessary $[7,19,20,48]$.

Imaging follow-up is more difficult for patients with CI. First, the magnet in the receiver coil leads to significant 


\section{Original article}
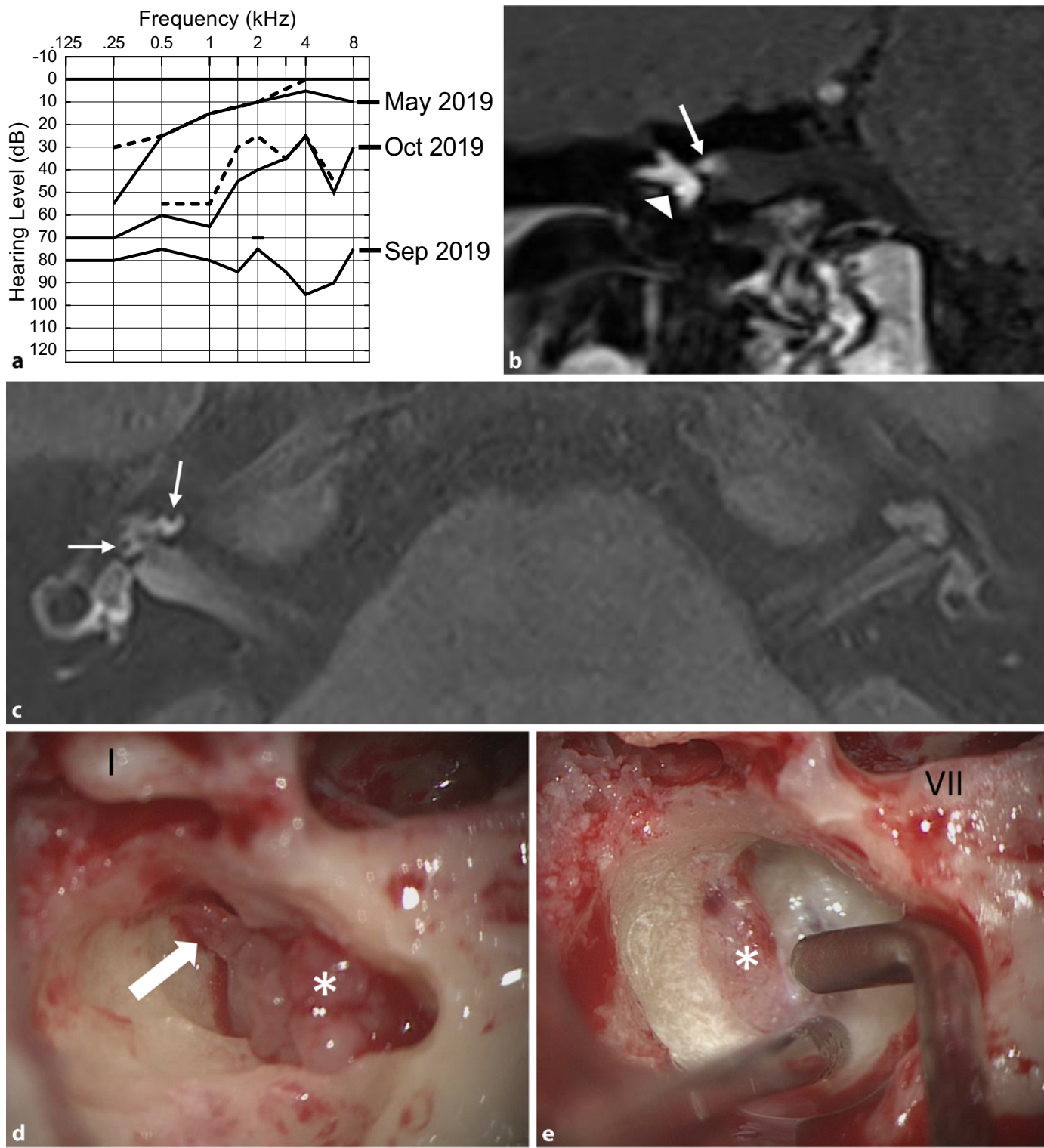

Fig. $5<$ Transmacular ISL (patient 8 in $\bullet$ Table 2). a Pure-tone audiogram showing fluctuating hearing loss (full line: air conduction, dashed line: bone conduction) most likely based on a secondary cochlear hydrops because of the tumor in the vestibule (c). b MRI (coronal, T1-w + Gd) showing the tumor in the right vestibule (triangle), the ampullary ends of the superior and lateral semicircular canals and extension along the superior vestibular nerve into the IAC $(\rightarrow)$. c MRI with hydrops sequence (3D inversion recovery, $6 \mathrm{~h}$ after systemic Gd application) showing a mild cochlear hydrops $(\rightarrow)$. d Intraoperative view with the tumor (asterisk) in the vestibule and along the superior vestibular nerve towards the fundus of the IAC $(\rightarrow)$. e-g The tumor (asterisk) is gradually separated from the facial nerve under facial nerve monitoring control. VII facial nerve, $G d$ gadolinium, / incus body, $w$ weighted 


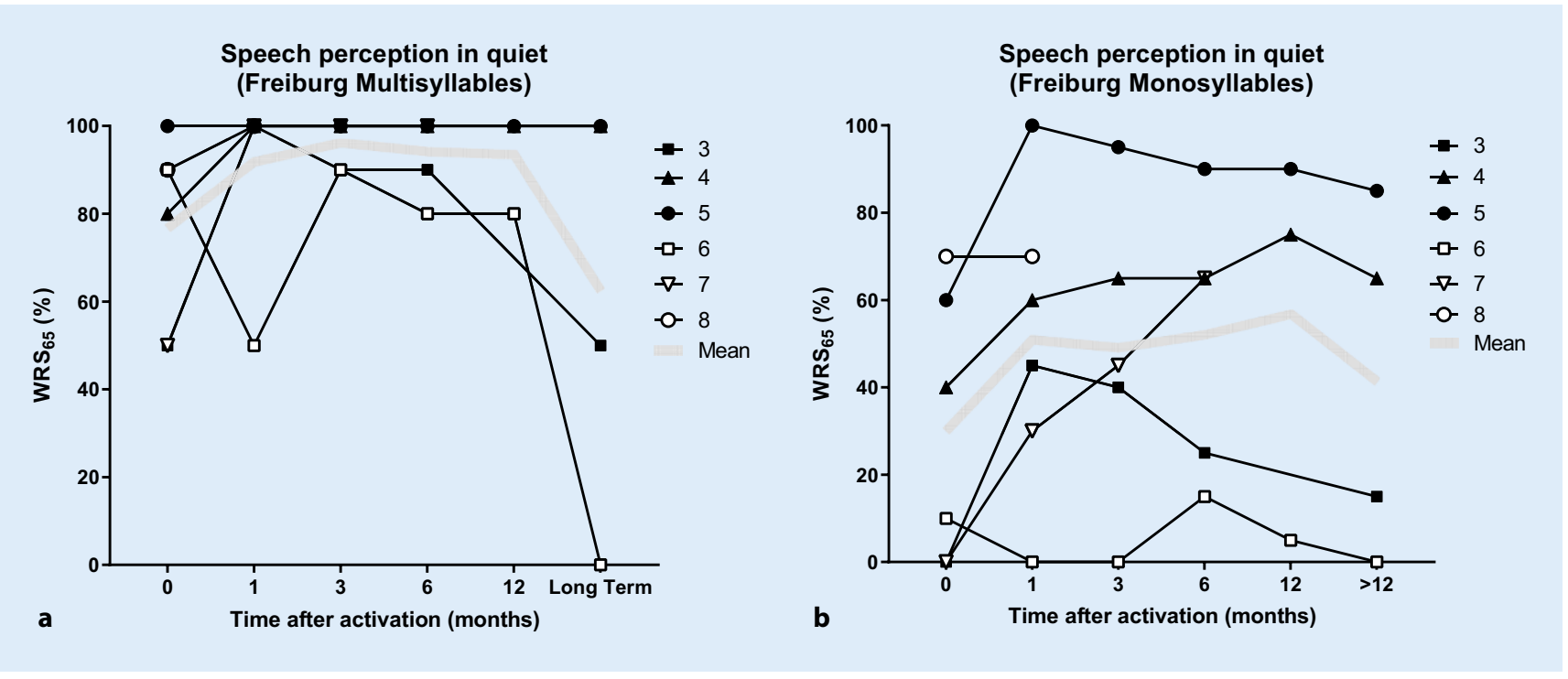

Fig. $6 \Delta$ Results of speech audiometry testing for a multisyllables (numbers) and b monosyllables with the cochlear implant in quiet at $65 \mathrm{~dB}$ SPL (WRS 65 ) as a function of time after activation of the speech processor
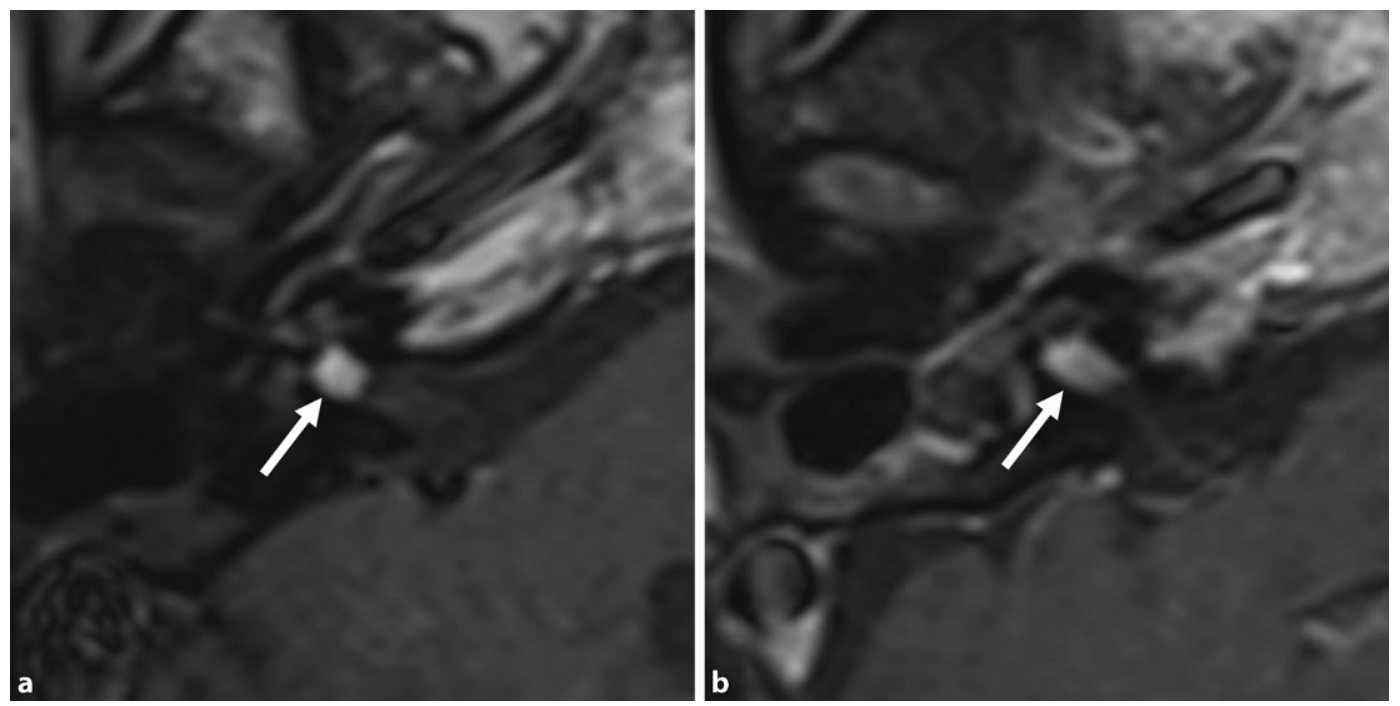

Fig. 7 A Postoperative MRI (axial, T1-w+Gd) for the two patients with poor hearing outcomes. a Patient 3, at 2 years after removal of the intracochlear tumor parts and Cl. Imaging of the IAC and the inner ear was possible by placement of the receiver coil of the Synchrony implant at a distance of approximately $9 \mathrm{~cm}$ from the outer ear canal. The tumor part in the fundus of the IAC had increased in size only very slightly by $0.5 \mathrm{~mm}$ (compare preoperative MRI: - Fig. 2e). b Patient 6, at 2 years and 5 months after removal of the intravestibulocochlear tumor parts and $\mathrm{Cl}$. Because of pain and heat development, the magnet of the $\mathrm{Cl} 512$ model had to be temporarily removed from the patient under local anesthesia. The MRI showed growth of the tumor parts in the IAC by $3.6 \mathrm{~mm}$ (compare preoperative MRI: - Fig. 4c). There was no tumor recurrence in the inner ear in the two patients. $C l$ cochlear implant, $w$ weighted, $\rightarrow$ tumor in the IAC

artefacts, which can impede the evaluation of the inner ear, the IAC, and the CPA. This problem can be counteracted by placement of the receiver coil at a distance of about $9-10 \mathrm{~cm}$ from the outer ear canal, which enables imaging of the inner ear and the IAC/CPA despite the implant (• Fig. 7a; [46, 49, 51]). Second, the magnetic field can induce significant heat and force, which may lead to pain and magnet dislocation, necessitating revision surgery [14, 23, 47, 49]. Temporary magnet removal or correct securing of a head splint can be used to overcome these problems. For the latter, standard operating procedures are available at our and many other institutions. Recently, magnets have been implemented in some implant models that align with the magnetic field, facilitating MRI follow-up [52, 53].

\section{Conclusion}

Cochleovestibular schwannomas with transmodiolar or transmacular extension are very rare and represent a special 
entity with respect to management. A therapeutic approach including incomplete and complete tumor removal requires a differentiated assessment considering the therapeutic goal and tumor location and extension. In addition, in these patients, CI can be an option for hearing rehabilitation.

\section{Corresponding address}

\section{Prof. Dr. med. S. K. Plontke}

Department of Otorhinolaryngology, Head \& Neck Surgery, Martin Luther University HalleWittenberg, University Medicine Halle Ernst-Grube-Str. 40, 06120 Halle (Saale), Germany stefan.plontke@uk-halle.de

Acknowledgments. The surgical videos (2D and 3D) were recorded with a fully digital microscope (ARRISCOPE). We thank Dr. Armin Schneider, Munich Surgical Imaging GmbH (formerly ARRI Medical $\mathrm{GmbH})$, Munich, Germany, for support with cutting and postproduction.

Funding. Open Access funding enabled and organized by Projekt DEAL.

\section{Compliance with ethical guidelines}

Conflict of interest. The authors declare the following competing interests:

S.K. Plontke: AudioCure Pharma GmbH, Berlin, Germany (consultant); MED-EL Austria and MED-EL Germany (travel reimbursement for speaking engagement); Oticon Medical, Denmark; Cochlear Ltd., Australia; Bundesministerium für Bildung und Forschung (research projects); BV-HNO e. V.; Merck Serono; Infectopharm; Dr.Willmar Schwabe GmbH \& Co. KG, Germany (lecture fees and travel reimbursement); P. Caye-Thomasen: Oticon Medical, Denmark; Cochlear Ltd., Australia (research projects); T. Rahne: Oticon Medical, Denmark; Cochlear Ltd., Australia (research projects), MED-EL Austria and MED-EL Germany (travel reimbursement for speaking engagement); G. Götze: MED-EL Austria und MED-EL Germany (research projects, travel reimbursement for speaking engagement). C. Strauss, S. Kösling, U. Siebolts, D. Vordermark, L. Wagner and L. Fröhlich declare that they have no competing interests.

Ethical standards. All procedures performed in studies involving human participants or on human tissue were in accordance with the ethical standards of the institutional and/or national research committee and with the 1975 Helsinki declaration and its later amendments or comparable ethical standards. Informed consent was obtained from all individual participants included in the study. Additional written informed consent was obtained from all individual participants or their legal representatives for whom identifying information is included in this article.
The supplement containing this article is not sponsored by industry.

Open Access. This article is licensed under a Creative Commons Attribution 4.0 International License, which permits use, sharing, adaptation, distribution and reproduction in any medium or format, as long as you give appropriate credit to the original author(s) and the source, provide a link to the Creative Commons licence, and indicate if changes were made. The images or other third party material in this article are included in the article's Creative Commons licence, unless indicated otherwise in a credit line to the material. If material is not included in the article's Creative Commons licence and your intended use is not permitted by statutory regulation or exceeds the permitted use, you will need to obtain permission directly from the copyright holder. To view a copy of this licence, visit http://creativecommons.org/licenses/by/4.0/.

\section{References}

1. Arndt S, Laszig R, Aschendorff A et al (2011) Unilateral deafness and cochlear implantation: audiological diagnostic evaluation and outcomes. HNO 59:437-446

2. Aschendorff A, Arndt S, Laszig R et al (2017) Treatment and auditory rehabilitation of intralabyrinthine schwannoma by means of cochlear implants: English version. HNO 65:46-51

3. Bartindale MR, Tadokoro KS, Kircher ML (2019) Cochlear implantation in sporadic vestibular schwannoma: a systematic literature review. JNeurol Surg B Skull Base 80:632-639

4. Bohr C, Muller S, Hornung J et al (2017) Hearing restoration with cochlear implants after translabyrinthine vestibular schwannoma resection. HNO 65:758-765

5. Borsetto D, Hammond-Kenny A, Tysome JR et al (2020) Hearing rehabilitation outcomes in cochlear implant recipients with vestibular schwannoma in observation or radiotherapy groups: a systematic review. Cochlear Implants Int 21:9-17

6. Carlson ML, NeffBA, Sladen DPetal (2016) Cochlear implantation in patients with intracochlear and iontralabyrinthine schwannomas. Otol Neurotol 37:647-653

7. Chen Z, Prasad SC, Di Lella F et al (2014) The behavior of residual tumors and facial nerve outcomes after incomplete excision of vestibular schwannomas. J Neurosurg 120:1278-1287

8. Choudhury B, Carlson ML, Jethanamest D (2019) Intralabyrinthine schwannomas: disease presentation, tumor management, and hearing rehabilitation. J Neurol Surg B Skull Base 80:196-202

9. Costello MS, Golub JS, Barrord JV et al (2016) Cochlear implantation after radiation therapy for acoustic neuroma. J Radiosurg SBRT 4:69-74

10. Dubernard X, Somers T, Veros Ket al (2014) Clinical presentation of intralabyrinthine schwannomas: a multicenter study of 110 cases. Otol Neurotol 35:1641-1649

11. Dziemba OC, Hocke T, Muller A et al (2018) Excitation characteristic of a bipolar stimulus for broadband stimulation in measurements of electrically evoked auditory potentials. Z Med Phys 28:73-77

12. Evans DG, Moran A, King A et al (2005) Incidence of vestibular schwannoma and neurofibromatosis 2 in the North West of England over a 10-year period: higher incidence than previously thought. Otol Neurotol 26:93-97

13. Firszt JB, Holden LK, Reeder RM et al (2012) Auditory abilities after cochlear implantation in adults with unilateral deafness: a pilot study. Otol Neurotol 33:1339-1346

14. Grupe G, Wagner J, Hofmann S et al (2017) Prevalence and complications of MRI scans of cochlear implant patients: English version. HNO 65:35-40

15. Hahn CH, Stangerup SE, Caye-Thomasen P (2013) Residual tumour after vestibular schwannoma surgery. J Laryngol Otol 127:568-573

16. Hansen MR, Gantz BJ, Dunn C (2013) Outcomes after cochlear implantation for patients with single-sided deafness, including those with recalcitrant Meniere's disease. Otol Neurotol 34:1681-1687

17. Hassepass F, Arndt S, Aschendorff A et al (2016) Cochlear implantation for hearing rehabilitation in single-sided deafness after translabyrinthine vestibular schwannoma surgery. Eur Arch Otorhinolaryngol 273:2373-2383

18. Hoppe U, Hocke T, Hast A et al (2019) Maximum preimplantation monosyllabic score as predictor of cochlear implant outcome. HNO. https://doi. org/10.1007/s00106-019-0648-0

19. Jacob JT, Carlson ML, Driscoll CL et al (2016) Volumetric analysis of tumor control following subtotal and near-total resection of vestibular schwannoma. Laryngoscope 126:1877-1882

20. KasbekarAV,Adan GH, Beacall Aetal (2018)Growth patterns of residual tumor in preoperatively growing vestibular schwannomas. J Neurol Surg B Skull Base 79:319-324

21. Kennedy RJ, Shelton C, Salzman KL et al (2004) Intralabyrinthine schwannomas: diagnosis, management, and a new classification system. Otol Neurotol 25:160-167

22. Klenzner T, Glaas M, Volpert S et al (2019) Cochlear implantation in patients with single-sided deafness after the translabyrinthine resection of the vestibular schwannoma-presented at the annual meeting of ADANO 2016 in Berlin. Otol Neurotol 40:e461-e466

23. Leinung $M$, Loth A, Groger M et al (2020) Cochlear implant magnet dislocation after MRI: surgical management and outcome. Eur Arch Otorhinolaryngol. https://doi.org/10.1007/ s00405-020-05826-x

24. Lenarz M, Sonmez H, Joseph G et al (2012) Cochlearimplantperformanceingeriatricpatients. Laryngoscope 122:1361-1365

25. Lloyd SKW, King AT, Rutherford SA et al (2017) Hearing optimisation in neurofibromatosis type 2: a systematic review. Clin Otolaryngol 42:1329-1337

26. Ma AK, Patel N (2020) Endoscope-assisted partial cochlectomy for intracochlear schwannoma with simultaneous cochlear implantation: a case report. Otol Neurotol 41:334-338

27. Merchant SN, Nadol JB (2010) Schwannoma. In: Merchant SN, Nadol JB (eds) Schuknecht's pathology of the ear. People's Medical Pubishing House PMPH, New Haven, CT, USA, pp 492-508

28. Morselli C, Boari N, Artico M et al (2020) The emerging role of hearing loss rehabilitation in patients with vestibular schwannoma treated with Gamma Knife radiosurgery: literature review. Neurosurg Rev. https://doi.org/10.1007/s10143020-01257-8

29. Nakatomi H, Jacob JT, Carlson ML et al (2017) Longterm risk of recurrence and regrowth after grosstotal and subtotal resection of sporadic vestibular 
schwannoma. J Neurosurg 19:1-7. https://doi. org/10.3171/2016.11.JNS16498

30. Patel KS, Ng E, KaurTetal (2019) Increased cochlear radiation dose predicts delayed hearing loss following both stereotactic radiosurgery and fractionated stereotactic radiotherapy for vestibular schwannoma. J Neurooncol 145:329-337

31. Pisa J, Sulkers J, Butler JB et al (2017) Stereotactic radiosurgery does not appear to impact cochlear implant performance in patients with neurofibromatosis type II. J Radiosurg SBRT 5:63-71

32. Plontke S, Lowenheim H, Preyer S et al (2005) Outcomes research analysis of continuous intratympanic glucocorticoid delivery in patients with acute severe to profound hearing loss: basis for planning randomized controlled trials. Acta Otolaryngol 125:830-839

33. Plontke SK (2020) An improved technique of subtotal cochleoectomy for removal of intracochlear schwannoma and single stage cochlear implantation. Otol Neurotol 41:e891. https://doi. org/10.1097/MAO.0000000000002718

34. Plontke S, Caye-Thomasen P, Strauss $C$ et al (2019) Aktuelle Aspekte zum Managment intralabyrinthärer Schwannome einschließlich subtotaler Cochleoektomie und Hörrehabilitation mittels Cochleaimplantat. Laryngo-Rhino-Otologie 98(S02):292-292. https://doi.org/10.1055/s0039-1686242

35. Plontke SK, Frohlich L, Wagner L et al (2020) How much cochlea do you need for cochlear implantation? Otol Neurotol 41:694-703

36. Plontke SK, Kosling S, Rahne T (2018) Cochlear implantation after partial or subtotal cochleoectomy for Intracochlear schwannoma removal-a technical report. Otol Neurotol 39:365-371

37. Plontke SK, Fröhlich L, Cozma Set al (2020) Hearing rehabilitation after subtotal cochleoectomy using a new, perimodiolar malleable cochlear implant electrode array: a preliminary report. Eur Arch Otorhinolaryngol. Epub ahead of print. https:// doi.org/10.1007/s00405-020-06098-1

38. Plontke SK, Rahne T, Pfister M et al (2017) Intralabyrinthine schwannomas : surgical management and hearing rehabilitation with cochlear implants. HNO 65:419-433

39. RahneT,HockeT,Strauss Cetal (2019) Perioperative recording of cochlear implant evoked brain stem responses after removal of the Intralabyrinthine portion of a vestibular schwannoma in a patient with NF2. Otol Neurotol 40:e20-e24

40. Rahne T, Plontke SK (2016) Functional result after cochlear implantation in children and adults with single-sided deafness. Otol Neurotol 37:e332-340

41. Reznitsky M, Petersen $M$, West $N$ et al (2019) Epidemiology of vestibular schwannomas-prospective 40-year data from an unselected national cohort. Clin Epidemiol 11:981-986

42. Roemer A, Lenarz T, Lesinski-Schiedat A (2017) Cochlear implantation improves hearing and vertigo in patients after removal of vestibular schwannoma. Int Tinnitus J 21:2-6

43. RohloffK, Koopmann M, Wei Det al (2017) Cochlear implantation in the elderly: does age matter? Otol Neurotol 38:54-59

44. Salzman KL, Childs AM, Davidson HC et al (2012) Intralabyrinthine schwannomas: imaging diagnosis and classification. AJNR Am J Neuroradiol 33:104-109

45. Sanna M, Medina MD, Macak A et al (2016) Vestibular schwannoma resection with ipsilateral simultaneous cochlear implantation in patients with normal contralateral hearing. Audio Neurootol 21:286-295

46. Schroder D, Grupe G, Rademacher G et a (2018) Magnetic resonance imaging artifacts and cochlear implant positioning at $1.5 \mathrm{~T}$ in vivo. Biomed Res Int 2018:9163285

47. Shew M, Wichova H, Lin J et al (2019) Magnetic resonance imaging with cochlear implants and auditory brainstem implants: are we truly practicing MRI safety? Laryngoscope 129:482-489

48. Syed MI, Wolf A, Ilan O et al (2017) The behaviour of residual tumour after the intentional incomplete excision of a vestibular schwannoma: is it such a bad thing to leave some behind? Clin Otolaryngol 42:92-97

49. Tam YC, Lee JWY, Gair J et al (2020) Performing MRI scans on cochlear implant and auditory brainstem implant recipients: review of 14.5 years experience. Otol Neurotol 41:e556-e562

50. Thompson NJ, O'connell BP, Brown KD (2019) Translabyrinthine excision of vestibular schwannoma with concurrent cochlear implantation: systematic review. J Neurol Surg B Skull Base 80:187-195

51. Todt I, Rademacher G, Mittmann P et al (2017) Postoperative imaging of the internal auditory canal : visualization of active auditory implants. HNO 65:81-86

52. Todt I, Tittel A, Ernst A et al (2017) Pain free $3 T$ MRI scans in cochlear Implantees. Otol Neurotol 38:e401-e404

53. Tysome JR, Tam YC, Patterson I et al (2019) Assessment of a novel 3T MRI compatible cochlear implant magnet: torque, forces, demagnetization, and imaging. Otol Neurotol 40:e966-e974

54. Van Abel KM, Carlson ML, Link MJ et al (2013) Primary inner ear schwannomas: a case series and systematic review of the literature. Laryngoscope 123:1957-1966

55. Vlastarakos PV, Nazos K, Tavoulari EF et al (2014) Cochlear implantation for single-sided deafness: the outcomes. An evidence-based approach. Eur Arch Otorhinolaryngol 271:2119-2126

56. Wagner L, Plontke SK, Fröhlich L et al (2020) Reduced spread of electric field after surgical removal of intracochlear schwannoma and cochlear implantation. Otol Neurotol (corrected proofs, in press)

57. West N, Sass H, Caye-Thomasen P (2020) Sporadic and NF2-associated vestibular schwannoma surgery and simultaneous cochlear implantation: a comparative systematic review. Eur Arch Otorhinolaryngol 277:333-342 